Published in final edited form as:

Sex Transm Dis. 2019 April ; 46(4): e32-e34. doi:10.1097/OLQ.0000000000000942.

\title{
Note: High interest in doxycycline for sexually transmitted infection post-exposure prophylaxis (doxycycline-PEP) in a multi-city survey of men who have sex with men (MSM) using a social-networking app
}

\author{
Matthew A. Spinelli, MD' ${ }^{1}$, Hyman M. Scott, MD $^{2}$, Eric Vittinghoff, PhD $^{3}$, Albert Y. Liu, MD², \\ Kenneth Coleman, $\mathbf{M A}^{2}$, and Susan P. Buchbinder, MD $^{2}$ \\ ${ }^{1}$ University of California, San Francisco, Division of HIV, ID, and Global Medicine \\ ${ }^{2}$ Bridge HIV, San Francisco Department of Public Health \\ ${ }^{3}$ University of California, San Francisco, Department of Epidemiology and Biostatistics
}

\begin{abstract}
Current strategies to prevent sexually-transmitted infections (STI) are not controlling the epidemic. Doxycycline STI post-exposure prophylaxis' efficacy (doxycycline-PEP) shows promise in pilot studies, but wider acceptability is unknown. A majority (84\%) of diverse individuals using a gay social-networking app were interested in doxycycline-PEP. Doxycycline-PEP should be examined in larger trials.
\end{abstract}

\section{Short summary:}

Sexually transmitted infections are increasing with new prevention options needed. A majority of MSM (84\%) using a gay social networking application were interested in using doxycycline STI prophylaxis.

\section{Keywords}

doxycycline post-exposure prophylaxis; sexually transmitted infection prophylaxis; men who have sex with men; social-networking applications; STI

\section{Introduction:}

Sexually-transmitted infections (STI) are increasing in both people living with HIV (PLWH) and HIV-uninfected men having sex with men (MSM). ${ }^{1,2}$ Increased detection through extragenital testing and screening programs linked to pre-exposure prophylaxis (PrEP) roll-out may contribute to this finding. ${ }^{2}$ However, there is also increased incidence of symptomatic syphilis and gonorrhea infections, suggesting that STI incidence is rising irrespective of increased detection of asymptomatic infections. ${ }^{1,2}$ Although condoms, counseling, and

\footnotetext{
`25 Van Ness Avenue, Suite 100 San Francisco, CA 94102, Phone: (415) 437-7415, Fax: (415) 476-9364, matthew.spinelli@ucsf.edu. Conflicts of Interest:The authors have no conflicts of interest to declare.
} 
screening continue to be cornerstones of STI prevention, condom use is falling and condoms may have limited real-world efficacy when used intermittently by MSM. ${ }^{3}$ Furthermore, STI screening in PrEP roll-out has been found to be sub-optimal so far. ${ }^{4}$ Other strategies may be needed to effectively control the STI epidemic.

Small, recently completed pilot trials of doxycycline prophylaxis for STIs suggest it could be efficacious among MSM. A recent pilot study of doxycycline prophylaxis in $30 \mathrm{PLWH}$ with prior syphilis found a reduction in a pooled outcome of bacterial STIs. ${ }^{5}$ A sub-study of the IPERGAY trial in France of doxycycline post-exposure prophylaxis (doxycycline-PEP) included $232 \mathrm{HIV}$-uninfected MSM, and found reduced hazard of syphilis and chlamydia, although minimal effect on gonorrhea; and was acceptable to study participants. ${ }^{6}$ Although these studies are promising, it is important to measure acceptability of prevention interventions in the populations that will use them prior to proceeding with larger trials. Little is known about the acceptability of doxycycline-PEP in larger, diverse, real-world populations at-risk of STIs. Additionally, willingness to participate in a placebo-controlled trial of doxycycline-PEP among the broader MSM population is unknown.

\section{Materials and Methods:}

We conducted an anonymous online survey of users of a gay social-networking app over two 24-hour periods in April 2018 in 6 US cities: Atlanta, Birmingham, Chicago, New York, San Francisco, and Seattle with an estimated 160,000 active users in those locations. We invited users to participate in the survey by broadcast message, which appeared when individuals opened the app. Users who agreed to participate were linked to an online survey.

We collected self-reported demographics, HIV status, PrEP use (current and past), sexual behaviors including condomless anal or vaginal/front-hole sex, diagnosis of a bacterial STI in the last year, and interest in using doxycycline-PEP. Participants were told that the pill was an antibiotic that was effective in preventing syphilis and chlamydia in a pilot study but had minimal effect on gonorrhea; and they would need to take doxycycline each time after sex. ${ }^{6}$ On the second survey distribution, respondents were also asked if they would be willing to participate in a study of doxycycline-PEP in which there was a 50\% chance of receiving a placebo. The survey took approximately 5 minutes to complete and participants could elect to be entered into a raffle to win a tablet computer.

Our study's aim was to measure reported interest in doxycycline-PEP in users of a gay social-networking app. In adjusted analysis using logistic regression, we examined factors associated with doxycycline-PEP interest.

All data were collected anonymously and the study was determined to be exempt by the University of California, San Francisco Institutional Review Board. Doxycycline is not approved for STI post-exposure prophylaxis by the FDA and its use for this indication is investigational. 


\section{Results:}

A total of 5,827 users clicked on the advertisement, and 1,301 respondents completed the survey (22\%). The median age was $34 ; 96 \%$ were cis-men, $1 \%$ transgender women, $1 \%$ transgender men, and $2 \%$ gender queer or non-binary. The sample was racially-ethnically diverse: 48\% White, 25\% Latinx, 16\% African-American, 6\% Asian, and 7\% Multiple/other. Overall, 16\% were people living with HIV (PLWH), 37\% were HIV-uninfected on PrEP, and $47 \%$ were HIV-infected not on PrEP. Most (80\%) reported condomless sex in the last 6 months, and 39\% reported receiving a diagnosis of a bacterial STI in the last year. Overall, $84 \%$ of participants expressed interest in trying doxycycline-PEP for prevention of STIs (Table). Of the participants who completed the second survey round ( $\mathrm{N}=480), 86 \%$ reported willingness to participate in a doxycycline-PEP study in which there was a 50\% chance of receiving a placebo.

The factors associated with higher interest in doxycycline-PEP (Table) included: AfricanAmerican race [AOR 1.8 (95\% confidence interval: 1.1-3.1)] and Latinx ethnicity [AOR 1.7 $(1.1-2.7)]$ vs. White race; reporting condomless sex [AOR 1.9 (1.3-2.7)] or having had a bacterial STI [AOR 1.6 (1.1-2.4)]. Interest did not differ when comparing PLWH, HIVnegative PrEP users, and HIV-negative individuals not using PrEP (heterogeneity $\mathrm{p}=0.86$ ).

\section{Discussion:}

In a large multi-city sample of individuals using a gay social networking app, respondents reported high interest in doxycycline-PEP for STI. Molina et al.'s study of doxycycline-PEP nested in the IPERGAY cohort reported high acceptance into the sub-study, with only about a fifth declining. ${ }^{6}$ Our study suggests that high acceptability of a doxycycline-PEP strategy extends outside of research settings, including in diverse patient populations in multiple U.S. cities.

The acceptability of doxycycline-PEP occurred in the context of more than a third of individuals having had an STI in the last year, and high prevalence of condomless sex. Individuals at higher risk of STI or with prior STI were more likely to express interest in doxycycline-PEP, with overall interest in at-risk populations quite high. Self-assessment of risk for STI may guide interest in doxycycline-PEP. However, many populations at-risk of HIV do not assess their risk accurately. ${ }^{7}$ It is possible that MSM may better assess STI risk due to high prevalence in the population, with individuals more likely to have experienced an STI in their lifetime.

A notable finding of our analysis is that African-American and Latinx respondents had higher interest in doxycycline-PEP than White respondents. The higher interest among these individuals may be related to the disproportionate impact of the STI epidemic in these populations. ${ }^{2,8}$ However, the disproportionate impact of the HIV epidemic has not led to higher PrEP interest, potentially due to the differential impact of structural barriers and/or stigma. ${ }^{9-11}$ Regardless of the mechanism, higher interest among Black and Latinx men could support uptake of STI prevention strategies in communities that could benefit most. Furthermore, the remarkably high overall interest and willingness to participate in a 
randomized placebo-controlled trial suggests that doxycycline-PEP research interventions could have high accrual and acceptability.

Limitations of this study include its cross-sectional design, limited generalizability to populations not using gay social-networking apps, few transgender individuals included in the sample, and the inability to correlate stated interest with later uptake into doxycyclinePEP research studies or STI interventions.

Doxycycline-PEP is a potential tool to address the STI epidemic in populations at-risk for STIs and is of remarkably high interest for individuals using a gay social-networking app. Future research will need to study actual doxycycline-PEP uptake and adherence in diverse patient populations, as well as measure doxycycline-PEP's safety, efficacy, and impact on antimicrobial resistance.

\section{Acknowledgments}

Support:This study was supported by National Institute of Health R01MH109320.

\section{References:}

1. An Q, Wejnert C, Bernstein K, Paz-Bailey G, Group NS. Syphilis Screening and Diagnosis Among Men Who Have Sex With Men, 2008-2014, 20 U.S. Cities. J Acquir Immune Defic Syndr. 2017;75 Suppl 3:S363-S369. [PubMed: 28604440]

2. Schillinger JA. The intersection of PrEP and Sexually Transmitted Infections. The Conference on Retroviruses and Opportunistic Infections; 2018; Boston.

3. Smith DK, Herbst JH, Zhang X, Rose CE. Condom effectiveness for HIV prevention by consistency of use among men who have sex with men in the United States. J Acquir Immune Defic Syndr. 2015;68(3):337-344. [PubMed: 25469526]

4. Spinelli MA, Scott HM, Vittinghoff E, et al. Provider Adherence to Pre-exposure Prophylaxis Monitoring Guidelines in a Large Primary Care Network. Open Forum Infect Dis. 2018;5(6).

5. Bolan RK, Beymer MR, Weiss RE, Flynn RP, Leibowitz AA, Klausner JD. Doxycycline prophylaxis to reduce incident syphilis among HIV-infected men who have sex with men who continue to engage in high-risk sex: a randomized, controlled pilot study. Sex Transm Dis. 2015;42(2):98-103. [PubMed: 25585069]

6. Molina JM, Charreau I, Chidiac C, et al. Post-exposure prophylaxis with doxycycline to prevent sexually transmitted infections in men who have sex with men: an open-label randomised substudy of the ANRS IPERGAY trial. Lancet Infect Dis. 2018;18(3):308-317. [PubMed: 29229440]

7. Serota DP, Rosenberg ES, Lockard AM, et al. Beyond the Biomedical: PrEP Failures in a Cohort of Young Black Men who have Sex with Men in Atlanta, GA. Clin Infect Dis. 2018.

8. Spinelli MA, Scott H, Vittinghoff E, et al. Factors impacting appropriate HIV/STI screening and PrEP persistence in primary care [\#1028]. Paper presented at: Conference on Retroviruses and Opportunistic Infections 2018; Boston.

9. Hoots BE, Finlayson T, Nerlander L, Paz-Bailey G, National HIVBSSG. Willingness to Take, Use of, and Indications for Pre-exposure Prophylaxis Among Men Who Have Sex With Men-20 US Cities, 2014. Clin Infect Dis. 2016;63(5):672-677. [PubMed: 27282710]

10. Franks J, Hirsch-Moverman Y, Loquere AS, et al. Sex, PrEP, and Stigma: Experiences with HIV Pre-exposure Prophylaxis Among New York City MSM Participating in the HPTN 067/ADAPT Study. AIDS Behav. 2018;22(4):1139-1149. [PubMed: 29143163]

11. Golub SA, Gamarel KE, Lelutiu-Weinberger C. The Importance of Sexual History Taking for PrEP Comprehension Among Young People of Color. AIDS Behav. 2017;21(5):1315-1324. [PubMed: 27475944] 
Table:

Factors associated with interest in doxycycline for STI post-exposure prophylaxis (doxycycline-PEP) and reported sexually-transmitted infection (STI) in the last year $(\mathrm{N}=1,301)$

\begin{tabular}{|c|c|c|}
\hline & $\begin{array}{c}\text { Overall } \\
(\mathbf{N}=1,301)\end{array}$ & $\begin{array}{l}\text { AOR for doxy-PEP interest } \\
\text { [95\% Confidence Interval (CI)] }\end{array}$ \\
\hline Age, median [inter-quartile range (IQR)] & $34(28-44)$ & $1.11(0.97-1.29)^{1}$ \\
\hline Race/ethnicity: White & $48 \%$ & Ref. \\
\hline African-American & $16 \%$ & $1.83(1.08-3.11)$ \\
\hline Asian & $6 \%$ & $1.09(0.57-2.09)$ \\
\hline Latinx & $25 \%$ & $1.74(1.14-2.65)$ \\
\hline Multiple/other & $7 \%$ & $0.90(0.50-1.62)$ \\
\hline Group: People Living with HIV & $16 \%$ & Ref. \\
\hline HIV-uninfected on PrEP & $37 \%$ & $0.86(0.51-1.46)$ \\
\hline HIV-uninfected not on PrEP & $47 \%$ & $0.89(0.52-1.50)$ \\
\hline Anal or vaginal/front-hole sex partners, median (IQR) & $5(2-15)$ & $0.98(0.95-1.01)^{2}$ \\
\hline Condomless sex last 6 months & $80 \%$ & $1.86(1.27-2.71)$ \\
\hline Bacterial STI last year & $39 \%$ & $1.64(1.13-2.39)$ \\
\hline City: San Francisco & $16 \%$ & Ref. \\
\hline Atlanta & $13 \%$ & $1.57(0.82-2.97)$ \\
\hline Birmingham & $1 \%$ & $1.11(0.30-4.11)$ \\
\hline Chicago & $16 \%$ & $1.20(0.68-2.10)$ \\
\hline New York City & $38 \%$ & $0.79(0.51-1.25)$ \\
\hline Seattle & $10 \%$ & $0.88(0.39-1.63)$ \\
\hline
\end{tabular}

AOR: adjusted odds ratio

${ }^{1}$ Scaled per ten years

${ }^{2}$ Scaled per 5 partners 